\title{
Low scale leptogenesis in a hybrid model of the scotogenic type I and III seesaw models
}

\author{
Daijiro Suematsu $\odot^{*}$ \\ Institute for Theoretical Physics, Kanazawa University, Kanazawa 920-1192, Japan
}

(Received 30 June 2019; published 10 September 2019)

\begin{abstract}
The scotogenic type I and type III seesaw models are good candidates to explain the existence of neutrino masses and dark matter simultaneously. However, since triplet fermions have $S U(2)$ gauge interaction, they cannot be out of equilibrium before the electroweak symmetry breaking. Thus, leptogenesis seems to be difficult within a framework of the pure type III seesaw model. Some extension seems to be required to solve this fault. A model extended by introducing a singlet fermion could be such a simple example. If the singlet fermion is in thermal equilibrium even for its extremely small neutrino Yukawa coupling, leptogenesis could be shown to occur successfully for a rather low mass of the singlet fermion. The required mass could be lowered to $10^{4} \mathrm{GeV}$.
\end{abstract}

DOI: $10.1103 /$ PhysRevD.100.055008

\section{INTRODUCTION}

Leptogenesis is considered to be the most promising scenario for the generation of baryon number asymmetry in the Universe [1,2]. In this scenario, lepton number asymmetry produced in some way is transformed into the baryon number asymmetry through the sphaleron interaction [3]. The lepton number asymmetry is usually considered to be caused through the decay of righthanded neutrinos which appear in the seesaw mechanism for the neutrino mass generation [4]. If the right-handed neutrinos have no interaction except for neutrino Yukawa couplings, both their production in the thermal plasma and their decay are brought about only through this interaction. If these couplings are strong, their production occurs effectively and they can reach equilibrium at an earlier stage. However, washout of the generated lepton number asymmetry is also caused by them effectively. On the other hand, if these couplings are weak, their production is ineffective and their equilibrium value is realized at a later stage although the washout effect could be suppressed. As a result, only a restricted range of the neutrino Yukawa couplings is expected to cause the required baryon number asymmetry via the leptogenesis successfully. This feature requires the mass of the right-handed neutrinos in the

\footnotetext{
*suematsu@hep.s.kanazawa-u.ac.jp
}

Published by the American Physical Society under the terms of the Creative Commons Attribution 4.0 International license. Further distribution of this work must maintain attribution to the author(s) and the published article's title, journal citation, and DOI. Funded by SCOAP. ordinary seesaw model to be more than $10^{9} \mathrm{GeV}$ [5] under the constraint of neutrino oscillation data as long as resonant leptogenesis [6] is not supposed. We find a similar feature in the scotogenic type I seesaw model [7], which is a well-known model for both neutrino masses and dark matter (DM) [8,9]. In this model, the righthanded neutrinos whose masses are in $\mathrm{TeV}$ ranges could have a chance to be a candidate for both DM and a mother field of leptogenesis [10].

The scotogenic type III seesaw model is known as another model which can connect the neutrino mass generation and the existence of DM at low energy regions [11]. It is a simple extension of the standard model (SM) by an additional inert doublet scalar $\eta$ and $S U$ (2) triplet fermions $\Sigma_{\alpha}\left(\alpha=1-n_{\Sigma}\right)$ which could play the same role as the right-handed neutrinos in the scotogenic type I seesaw model. If odd parity of a $Z_{2}$ symmetry imposed on the model is assigned to these new fields and all other fields are assumed to have its even parity, the neutrino masses are forbidden at tree level but they are generated through a one-loop diagram. This model can have also two DM candidates, a neutral component of $\eta$ and the lightest neutral one of $\Sigma_{\alpha}$, whose stability is guaranteed by the $Z_{2}$ symmetry. In both cases, one might expect that the decay of the lightest or next lightest triplet fermion causes the lepton number asymmetry, depending on which is the DM, since it violates the lepton number. However, it is difficult for this decay to cause a net lepton number asymmetry unfortunately since $\Sigma_{\alpha}$ are considered to have masses near the TeV ranges. Since the triplet fermions $\Sigma_{\alpha}$ have $S U(2)$ gauge interaction differently from the right-handed neutrino, it cannot be out of equilibrium before the 
electroweak symmetry breaking [12]. ${ }^{1}$ In that case, their decay cannot satisfy the Sakharov conditions for the generation of the lepton number asymmetry. In this paper, we try to extend the scotogenic type III seesaw model to incorporate the leptogenesis into it in a self-contained way, assuming that the $\Sigma_{\alpha}$ mass is much smaller than $O\left(10^{9}\right) \mathrm{GeV}$. In that extension, the sufficient baryon number asymmetry is found to be produced by a mother fermion with a mass of $O\left(10^{4}\right) \mathrm{GeV}$.

The paper is organized as follows. In the next section, we introduce a scotogenic type III seesaw model and give a brief review of the neutrino mass generation and the DM abundance in it. After that, its extension is discussed by introducing a singlet fermion and we address how it makes leptogenesis possible. In Sec. III, the leptogenesis is studied quantitatively to show that it could occur for a rather low mass mother fermion. The paper is summarized in Sec. IV.

\section{A HYBRID SCOTOGENIC MODEL}

\section{A. Neutrino mass and DM abundance in scotogenic type III seesaw}

The scotogenic type III seesaw model [11] is characterized by the neutrino Yukawa couplings of $S U(2)$ triplet fermions $\Sigma_{\alpha}$ with a hypercharge $Y=0$ and an inert doublet scalar $\eta$ with $Y=-1$, which are given as

$-\mathcal{L}_{\Sigma}=\sum_{\alpha=1}^{n_{\Sigma}}\left(\sum_{i=e, \mu, \tau} h_{i \alpha} \bar{\ell}_{L_{i}} \Sigma_{\alpha} \eta+\frac{1}{2} M_{\alpha} \operatorname{tr}\left(\bar{\Sigma}_{\alpha} \Sigma_{\alpha}^{c}\right)+\right.$ H.c. $)$,

where $\Sigma_{\alpha}$ is defined by

$$
\Sigma_{\alpha} \equiv \sum_{a=1}^{3} \frac{\tau^{a}}{2} \Sigma_{\alpha}^{a}=\frac{1}{2}\left(\begin{array}{cc}
\Sigma_{\alpha}^{0} & \sqrt{2} \Sigma_{\alpha}^{+} \\
\sqrt{2} \Sigma_{\alpha}^{-} & -\Sigma_{\alpha}^{0}
\end{array}\right) .
$$

The scalar potential of the model is given by

$$
\begin{aligned}
V= & m_{\phi}^{2} \phi^{\dagger} \phi+m_{\eta}^{2} \eta^{\dagger} \eta+\lambda_{1}\left(\phi^{\dagger} \phi\right)^{2}+\lambda_{2}\left(\eta^{\dagger} \eta\right)^{2} \\
& +\lambda_{3}\left(\phi^{\dagger} \phi\right)\left(\eta^{\dagger} \eta\right)+\lambda_{4}\left(\phi^{\dagger} \eta\right)\left(\eta^{\dagger} \phi\right) \\
& +\frac{\lambda_{5}}{2}\left[\left(\eta^{\dagger} \phi\right)^{2}+\left(\phi^{\dagger} \eta\right)^{2}\right],
\end{aligned}
$$

where $\phi$ is an ordinary Higgs doublet scalar. Since we impose a $Z_{2}$ symmetry for which only $\Sigma_{\alpha}$ and $\eta$ have odd parity and all other fields are assigned even parity, their allowed interaction terms except for gauge interactions are restricted to the ones listed in Eqs. (1) and (3).

\footnotetext{
${ }^{1}$ Leptogenesis in the type III seesaw model has been studied in [13]. It has been shown that the sufficient lepton number asymmetry can be generated as long as the mass of the mother triplet fermion is larger than $O\left(10^{9}\right) \mathrm{GeV}$. However, it has also been discussed that successful leptogenesis is not so easy for a much lighter triplet fermion.
}
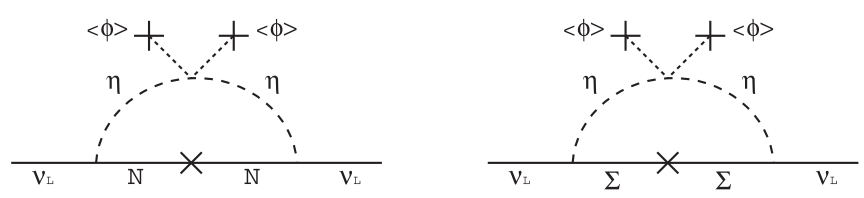

FIG. 1. Left: A one-loop diagram for the neutrino mass generation in the scotogenic type I seesaw. Right: A one-loop diagram for the neutrino mass generation in the scotogenic type III seesaw.

This $Z_{2}$ symmetry brings about interesting features in the model. Since $\eta$ is assumed to have no vacuum expectation value, the $Z_{2}$ symmetry remains as an exact one. Thus, the neutrinos cannot have masses at a tree level. However, as the scotogenic type I seesaw model shown in the left of Fig. 1, the neutrino masses are generated by a one-loop diagram shown in the right of Fig. 1, in which the righthanded neutrino $N$ in the former is replaced by $\Sigma_{\alpha}$. The mass induced through this diagram is estimated as

$\mathcal{M}_{i j}=\sum_{\alpha=1}^{n_{\Sigma}} \frac{h_{i \alpha} h_{j \alpha} \lambda_{5}\langle\phi\rangle^{2}}{32 \pi^{2} M_{\alpha}}\left[\frac{M_{\alpha}^{2}}{M_{\eta}^{2}-M_{\alpha}^{2}}\left(1+\frac{M_{\alpha}^{2}}{M_{\eta}^{2}-M_{\alpha}^{2}}\right) \ln \frac{M_{\alpha}^{2}}{M_{\eta}^{2}}\right]$,

where $M_{\eta}^{2}=m_{\eta}^{2}+\left(\lambda_{3}+\lambda_{4}\right)\langle\phi\rangle^{2}$. If we note that only two triplet fermions are enough to explain the neutrino oscillation data, Yukawa coupling constants for the remaining ones can be very small so as not to contribute to the neutrino mass generation substantially. Taking this into account, we confine our study here to the minimal case $n_{\Sigma}=2$. An interesting feature of the model is that both $M_{\alpha}$ and $M_{\eta}$ can take much smaller values in comparison with typical ones for the right-handed neutrino masses in the ordinary type I seesaw model as long as $\left|\lambda_{5}\right| \ll 1$ is satisfied.

Another interesting feature is that the model could explain a required value of the DM abundance. The model has two DM candidates as mentioned above, that is, the lightest $\Sigma_{\alpha}^{0}$ and the lightest neutral component of $\eta$. Both of them have the $Z_{2}$ odd parity. In this paper, we focus our study on a case where $\eta$ is DM. ${ }^{2}$ This DM candidate has been extensively studied in many articles $[7,15]$. There, it has been proved that the lightest neutral component of $\eta$ with the mass of $O(1) \mathrm{TeV}$ can realize the required DM relic density easily. In fact, since the coannihilation among the components of $\eta$ could be effective, $\Omega h^{2}=0.12$ can be obtained for suitable values of quartic couplings $\lambda_{3}$ and $\lambda_{4}$ without serious fine tuning. In the following discussion, we just assume $M_{\eta}=O(1) \mathrm{TeV}$, which can guarantee the $\mathrm{DM}$ abundance.

These are common features to the scotogenic type I seesaw model. However, a problem is caused in the leptogenesis through the nature of $\Sigma_{\alpha}$, which has $S U(2)$

\footnotetext{
${ }^{2}$ The study of a case in which $\Sigma^{0}$ is DM can be found in $[11,14]$.
} 
gauge interactions other than the neutrino Yukawa couplings given in Eq. (1). As a result, the $\Sigma_{\alpha}$ decay cannot generate the lepton number asymmetry differently from the right-handed neutrino decay in the scotogenic type I seesaw model. This is because they cannot leave thermal equilibrium until a scale of the electroweak symmetry breaking as noted before. In order to remedy this fault and make the leptogenesis available in this framework, we have to consider some extension of the model.

\section{B. A simple extension of the model}

As a simple extension, ${ }^{3}$ we consider introducing a $Z_{2}$ odd singlet fermion $N$ and adding several new terms to the Lagrangian given in Eq. (1) such that ${ }^{4}$

$$
\begin{aligned}
-\mathcal{L}_{N}= & \sum_{i=e, \mu, \tau} h_{i}^{N} \bar{\ell}_{L_{i}} N \eta+\frac{1}{2} M_{N} \bar{N} N^{c}+\frac{1}{2} y_{N} S \bar{N} N^{c} \\
& +\sum_{\alpha=1}^{2} \frac{1}{2} y_{\alpha} \operatorname{tr}\left(\bar{\Sigma}_{\alpha} \Sigma_{\alpha}^{c}\right) S+\text { H.c. }
\end{aligned}
$$

where $S$ is a $Z_{2}$ even real scalar which has potential $\frac{1}{4} \lambda_{S} S^{4}+\frac{1}{2} m_{S}^{2} S^{2}$. The mass $m_{S}$ is assumed to satisfy $m_{S} \gg M_{\alpha}>M_{N}$. This model can be considered as a hybrid model of the two types of scotogenic model since the neutrino masses could be generated through the two types of diagram given in Fig. 1. However, if the coupling constant $h_{i}^{N}$ is sufficiently small, the neutrino mass formula (4) is not affected by this extension. On the other hand, the smallness of Yukawa coupling $h_{i}^{N}$ could make the substantial $N$ decay start at a low temperature such as $T \ll M_{N}$, where $\Gamma_{N} \gtrsim H$ is realized for the $N$ decay width $\Gamma_{N}=\sum_{i} \frac{h_{i}^{N 2}}{8 \pi} M_{N}$ and the Hubble parameter $H^{2}=\frac{\frac{\pi^{2}}{30} g_{*} T^{4}}{3 M_{p l}^{2}}$. The decay before reaching this temperature region is out of equilibrium. Thus, as long as $N$ has already been in thermal equilibrium at a high temperature $T>M_{N}$ through a certain interaction, it could generate the lepton number asymmetry efficiently.

At first, we address how $N$ could be in thermal equilibrium in such a case that its Yukawa couplings $h_{i}^{N}$ are very small. We suppose that $S$ has a nonminimal coupling with a Ricci scalar such as $\frac{\xi}{2} S^{2} R$. In that case, $S$ could play the role of the inflaton in the same way as Higgs inflation [17,18]. This inflation is expected to explain the present observational data for the CMB well for appropriate values of $\lambda_{S}$ and $\xi$. Since its details are not crucial for the present purpose, we confine the present discussion to the estimation of reheating temperature only. ${ }^{5}$

\footnotetext{
${ }^{3}$ The hybrid model of type I and type III is considered in a different context [16].

${ }^{4}$ Although masses of $\Sigma_{\alpha}$ and $N$ could be supposed to be generated by a vacuum expectation value of $S$ [12], they are assumed to be independent parameters for simplicity in this study.

${ }^{5}$ Several inflation scenarios have been discussed in the scotogenic type I seesaw model extended by a singlet scalar [19].
}

The reheating is dominantly caused by the $S$ decay to $\Sigma_{1.2}$ pairs through the couplings in Eq. (5) in the case $y_{1,2}>y_{N}$. There, the reheating temperature can be estimated from $H \simeq \Gamma_{S}^{D}$ by using both the Hubble parameter $H$ and the decay width $\Gamma_{S}^{D}$ of $S$ such as

$$
T_{R} \simeq 4 \times 10^{11}\left(\frac{y_{\Sigma}}{10^{-2}}\right)\left(\frac{m_{S}}{10^{10} \mathrm{GeV}}\right)^{1 / 2} \mathrm{GeV},
$$

where $y_{1,2}=y_{\Sigma}$ is assumed and $g_{*}=121.5$ is used for relativistic degrees of freedom in the model. Here, it is important to note that $N$ is pair-produced in the thermal plasma through the scattering of $\Sigma_{1,2}$ pairs mediated by $S$ even if the Yukawa coupling constants $h_{i}^{N}$ are sufficiently small. In that case, the Yukawa coupling constants $h_{i}^{N}$ could be irrelevant to the determination of the abundance of $N$. This is a completely different situation from the pure scotogenic type I seesaw case [7]. On the other hand, using the assumption $\frac{m_{S}}{2}>M_{1,2}>M_{N}$, we can roughly estimate the freeze-out temperature of this scattering process from $H \simeq \Gamma_{\Sigma_{\alpha} \Sigma_{\alpha} \rightarrow N N}^{S}$, where $\Gamma_{\Sigma_{\alpha} \Sigma_{\alpha} \rightarrow N N}^{S}$ is the reaction rate for $\Sigma_{\alpha} \Sigma_{\alpha} \rightarrow N N$, such that

$T_{D} \simeq 2 \times 10^{10}\left(\frac{10^{-2}}{y_{\Sigma}}\right)^{2 / 3}\left(\frac{10^{-2}}{y_{N}}\right)^{2 / 3}\left(\frac{m_{S}}{10^{10} \mathrm{GeV}}\right)^{4 / 3} \mathrm{GeV}$.

Equations (6) and (7) suggest that $N$ could reach thermal equilibrium at a certain temperature $T$ such that $T_{D}<T<T_{R}$. After that, it decouples from the thermal plasma at $T<T_{D}$ and starts the out-of-equilibrium decay to $\ell \eta^{\dagger}$ to generate the lepton number asymmetry.

In order to confirm that this scenario works, as an example, we fix the relevant parameters as follows:

$$
\begin{aligned}
y_{\Sigma} & =10^{-1.5}, \quad y_{N}=10^{-2}, \\
h_{i}^{N} & =10^{-6}, \quad m_{S}=10^{10} \mathrm{GeV}, \\
M_{1} & =10^{7} \mathrm{GeV}, \quad M_{2}=10^{8} \mathrm{GeV}, \\
M_{N} & =10^{6} \mathrm{GeV} .
\end{aligned}
$$

For these parameters, the reheating temperature obtained through the $S$ decay can be estimated as $T_{R} \simeq$ $4 \times 10^{11} \mathrm{GeV}$ from Eq. (6). To examine the evolution of the number density of $N$, we solve the Boltzmann equation for the number density of $N$ [20],

$\frac{d Y_{N}}{d z}=-\frac{z}{s H\left(M_{N}\right)}\left(\frac{Y_{N}}{Y_{N}^{\mathrm{eq}}}-1\right)\left\{\gamma_{D}+\left(\frac{Y_{N}}{Y_{N}^{\mathrm{eq}}}+1\right) \gamma_{\Sigma \Sigma}\right\}$,

where $\Sigma_{1,2}$ are supposed to be in thermal equilibrium; $z$ and $H\left(M_{N}\right)$ are defined as $z \equiv \frac{M_{N}}{T}$ and $H\left(M_{N}\right) \equiv 0.33 g_{*}^{1 / 2} \frac{M_{N}^{2}}{M_{\mathrm{pl}}}$; 

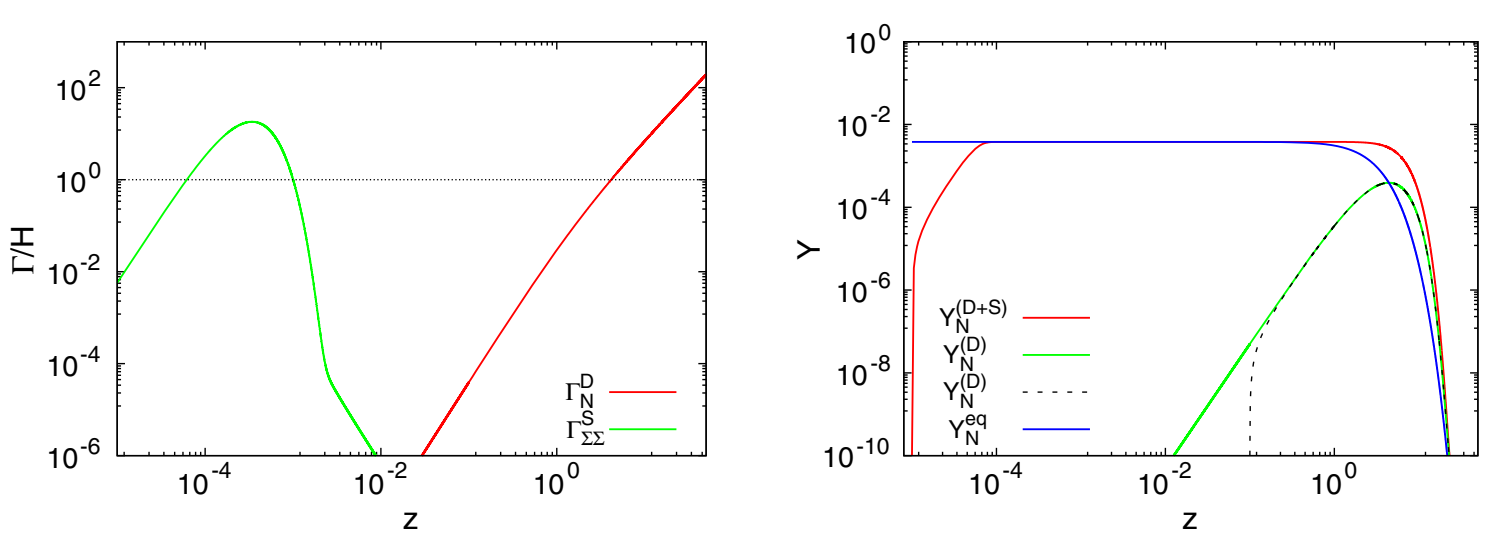

FIG. 2. The left panel shows the reaction rate $\Gamma$ normalized by the Hubble parameter $H$ which is relevant to the $N$ production, that is, the 2-2 scattering $\Sigma_{\alpha} \Sigma_{\alpha} \rightarrow N N\left(\Gamma_{\Sigma \Sigma}^{S}\right)$ and the decay $N \rightarrow \ell \eta^{\dagger}\left(\Gamma_{N}^{D}\right)$. The right panel shows the evolution of $Y_{N}$ for an initial condition $Y_{N}\left(z_{i}\right)=0$ with $z_{i}=z_{R}\left(\equiv \frac{M_{N}}{T_{R}}\right) . Y_{N}^{\mathrm{eq}}$ represents the thermal equilibrium value. Both $\gamma_{D}$ and $\gamma_{\Sigma \Sigma}$ are taken into account in $Y_{N}^{(D+S)}$ but only $\gamma_{D}$ is taken into account in $Y_{N}^{(D)}$. As a reference, $Y_{N}^{(D)}$ is plotted for the case $z_{i}=0.1$ using a black dashed line.

$Y_{N}$ is defined as $Y_{N} \equiv \frac{n_{N}}{s}$ by using the $N$ number density $n_{N}$ and the entropy density $s ; Y_{N}^{\mathrm{eq}}$ represents its equilibrium value; and $\gamma_{D}$ and $\gamma_{\Sigma \Sigma}$ stand for the reaction density of the $N$ decay and the 2-2 scattering $\Sigma_{\alpha} \Sigma_{\alpha} \rightarrow N N$, respectively [21].

The solution of Eq. (9) is plotted in Fig. 2. In the left panel, the ratio of each reaction rate to the Hubble parameter $\frac{\Gamma}{H}$ is plotted as a function of $z$. They are relevant to the production of $N$. In the right panel, the evolution of $Y_{N}$ is plotted as a function of $z$ for two cases, that is, in $Y_{N}^{(D+S)}$ where both the inverse decay of $N$ and the 2-2 scattering of a $\Sigma_{\alpha}$ pair are taken into account, but in $Y_{N}^{(D)}$ where the former is taken into account alone. The comparison of both panels suggests that the thermal equilibrium abundance of $N$ is realized when the 2-2 scattering reaches equilibrium, and it is kept still after the 2-2 scattering leaves equilibrium. The out-of-equilibrium decay of $N$ starts at $z>1$. Since $Y_{N}\left(z_{R}\right)=0$ is assumed as an initial value at $T_{R}$, the right panel shows that $N$ is efficiently produced by the 2-2 scattering and $Y_{N}^{(D+S)}$ reaches the equilibrium value $Y_{N}^{\mathrm{eq}}$ at a higher temperature compared with no scattering one $Y_{N}^{(D)}$. We can also find from this panel that the out-ofequilibrium decay of $N$ could start at a larger $Y_{N}^{\mathrm{eq}}$ value in the $Y_{N}^{(D+S)}$ case than the one in the $Y_{N}^{(D)}$ case. The difference is found to be 1 order of magnitude in this example. This feature does not depend on $T_{R}$ as long as $Y_{N}$ reaches its equilibrium value before $z \sim 1$. It suggests that $N$ could be a good mother fermion for the lepton number asymmetry in this extended model. Since both the mass and the couplings of $N$ are free from the neutrino mass constraint, a window might be opened for the low scale leptogenesis.

In the same panel, as a reference, $Y_{N}$ is plotted also for the case $Y_{N}^{(D)}(0.1)=0$ by a dashed line. It shows that $Y_{N}^{(D)}$ immediately reaches the same value for the case $Y_{N}^{(D)}\left(z_{R}\right)=0$. This suggests that we can take a much larger $z$ than $z_{R}$ as a starting point for the analysis of the
Boltzmann equation. Taking this into account, we discuss the possibility of a low scale leptogenesis caused by the $N$ decay quantitatively in the next section.

\section{LEPTOGENESIS}

The $N$ decay could satisfy the Sakharov condition and then it generates the lepton number asymmetry, which is converted to the baryon number asymmetry through the sphaleron process. If the sphaleron is in thermal equilibrium, the baryon number $B$ is found to be related with $B-L$ as $B=\frac{8}{23}(B-L)$ in the present model by using the chemical equilibrium condition [22]. If we use this relation for $Y_{B}$ and $Y_{B-L}$, which are defined as $Y_{B} \equiv \frac{n_{B}}{s}$ and $Y_{B-L} \equiv \frac{n_{B}-n_{L}}{s}$, by using the entropy density $s, Y_{B}$ in the present Universe is found to be obtained from $Y_{B-L}$, which is produced through $N$ decay as

$$
Y_{B}=\frac{8}{23} Y_{B-L}\left(z_{E W}\right),
$$

where the dimensionless parameter $z$ is defined in the previous part and $z_{E W}$ is fixed by the sphaleron decoupling temperature $T_{E W}$ as $z_{E W}=\frac{M_{N}}{T_{E W}}$.

The $C P$ asymmetry in the $N$ decay is dominantly caused by the interference between a tree diagram and a one-loop vertex diagram which has $\Sigma_{\alpha}$ in an internal line. They are shown in Fig. 3. It is calculated as [23]

$$
\begin{aligned}
\varepsilon \equiv & \frac{\Gamma\left(N \rightarrow \ell \eta^{\dagger}\right)-\Gamma\left(N^{c} \rightarrow \bar{\ell} \eta\right)}{\Gamma\left(N \rightarrow \ell \eta^{\dagger}\right)+\Gamma\left(N^{c} \rightarrow \bar{\ell} \eta\right)} \\
= & \frac{3}{64 \pi\left[\frac{3}{4}+\frac{1}{4}\left(1-\frac{M_{\eta}^{2}}{M_{N}^{2}}\right)^{2}\right]} \\
& \times \sum_{\alpha=1,2} \frac{\operatorname{Im}\left[\left(\sum_{i=e, \mu, \tau} h_{i}^{N} h_{i \alpha}^{*}\right)^{2}\right]}{\sum_{i=e, \mu, \tau} h_{i}^{N} h_{i}^{N *}} G\left(\frac{M_{\alpha}^{2}}{M_{N}^{2}}, \frac{M_{\eta}^{2}}{M_{N}^{2}}\right),
\end{aligned}
$$

where $G(x, y)$ is defined as 


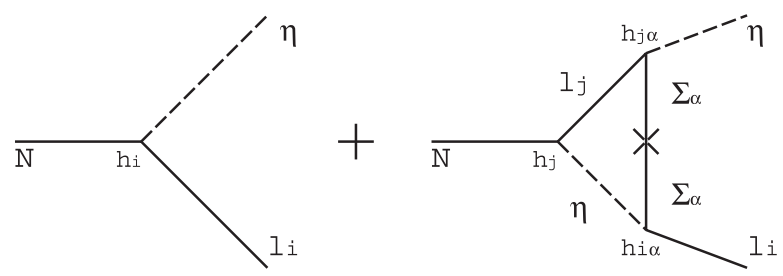

FIG. 3. Diagrams of the lepton number violating $N$ decay. The $C P$ asymmetry is induced by the interference between a tree and a one-loop diagram.

$$
\begin{aligned}
G(x, y)= & \frac{5}{4} F(x, 0)+\frac{1}{4} F(x, y) \\
& +\frac{1}{4}(1-y)^{2}[F(x, 0)+F(x, y)], \\
F(x, y)= & \sqrt{x}\left[1-y-(1+x) \ln \left(\frac{1-y+x}{x}\right)\right] .
\end{aligned}
$$

In order to estimate the lepton number asymmetry quantitatively, we need to fix a flavor structure of neutrino Yukawa coupling constants $h_{\alpha i}$ and $h_{i}^{N}$. ${ }^{6}$ Here, we adopt the tri-bimaximal flavor structure as an example. In the previous works [7], we find that tri-bimaximal flavor structure does not cause a serious effect in the study of leptogenesis compared with the one where nonzero $\theta_{13}$ is taken into account. ${ }^{7}$ We assume [25]

$h_{e 1}=0, \quad h_{\nu 1}=h_{\tau 1} \equiv h_{1}$;

$h_{e 2}=h_{\mu 2}=-h_{\tau 2} \equiv h_{2} ; \quad h_{e}^{N}=0, \quad h_{\mu}^{N}=h_{\tau}^{N} \equiv h_{N}$,

where $h_{i}^{N}$ is taken to be very small so that it is irrelevant to the neutrino mass and mixing. By using this flavor structure of the neutrino Yukawa couplings, $\varepsilon$ is found to be expressed as

$$
\varepsilon=\frac{3\left|h_{1}\right|^{2}}{32 \pi} G\left(\frac{M_{1}^{2}}{M_{N}^{2}}, \frac{M_{\eta}^{2}}{M_{N}^{2}}\right) \sin \left(2 \varphi_{1}\right)
$$

where $\varphi_{1}=\arg \left(h_{N}\right)-\arg \left(h_{1}\right)$. Here, we should note that $\varepsilon$ could take a larger value compared with the one in the pure scotogenic type I seesaw model since a singlet fermion is replaced by a triplet fermion $\Sigma_{\alpha}$ in an internal line of the one-loop diagram.

\footnotetext{
${ }^{6}$ As stressed in [24], $\varepsilon$ does not depend on the PontecorvoMaki-Nakagawa-Sakata (PMNS) matrix. However, the PMNS matrix could affect the reaction rate of the processes which contribute to the washout of the generated lepton number asymmetry.

${ }^{7}$ Although the model is different from the one studied in [7], the neutrino mass generation is the same except that $N$ is replaced by $\Sigma^{0}$ as shown in Fig. 1. Since nonzero $\theta_{13}$ effects on the neutrino Yukawa couplings are considered to appear in both models in the same way, we can follow the results there.
}

If $N$ is in thermal equilibrium, the substantial generation of the lepton number asymmetry is expected to start at $z \sim 1$, where $N$ leaves equilibrium as found in the right panel of Fig. 2. Thus, $Y_{B-L}\left(z_{E W}\right)$ might be roughly estimated as $Y_{B-L}\left(z_{E W}\right) \simeq \varepsilon \kappa Y_{N}^{\mathrm{eq}}(1)$ by using the equilibrium expression $Y_{N}^{\mathrm{eq}}(z)=\frac{45}{2 \pi^{4} g_{*}} z^{2} K_{2}(z)$, where $g_{*}$ is the number of relativistic degrees of freedom at this period and $K_{2}(z)$ is the modified Bessel function of the second kind. $\kappa$ stands for the washout efficiency for the generated lepton number asymmetry. Since the present value of $Y_{B}$ [26] requires $2.4 \times 10^{-10}<\left|Y_{B-L}\left(z_{E W}\right)\right|<2.7 \times 10^{-10}$, we find that $\varepsilon$ has to satisfy $|\varepsilon| \gtrsim 8 \times 10^{-8} \kappa^{-1}$ from this rough estimation. In the case of $M_{1}>M_{N}$, this $\varepsilon$ value requires

$\left|h_{1}\right|>8.5 \times 10^{-4}\left(\frac{M_{1}}{10^{7} \mathrm{GeV}}\right)^{1 / 2}\left(\frac{10^{6} \mathrm{GeV}}{M_{N}}\right)^{1 / 2}$

if $\varphi_{1}=\frac{\pi}{4}$ is assumed. If the out-of-equilibrium decay of $N$ starts at $z \sim 1$, its decay width $\Gamma_{N}^{D}$ should satisfy $H>\Gamma_{N}^{D}$ there. On the other hand, its decay should be completed at some $z_{T}$ before reaching the sphaleron decoupling temperature $T_{E W} \sim 100 \mathrm{GeV}$ and then $\left.H\right|_{z=z_{E W}}<\Gamma_{N}^{D}$ should be satisfied. In such a case, $Y_{N}$ can take its equilibrium value at $z>z_{T}$. These impose the condition

$6.2 \times 10^{-10}\left(\frac{10^{6} \mathrm{GeV}}{M_{N}}\right)^{1 / 2}<h_{N}<6.2 \times 10^{-6}\left(\frac{M_{N}}{10^{6} \mathrm{GeV}}\right)^{1 / 2}$.

This suggests that a favored range of $h_{N}$ becomes narrower for a smaller value of $M_{N}$.

The situation is completely different from the case discussed above, if $N$ has to be produced only through the neutrino Yukawa coupling $h_{N}$ from an initial value $Y_{N}\left(z_{R}\right)=0$. Since the inverse decay rate of $N$, which is a dominant process of the $N$ production, is proportional to $h_{N}^{2} M_{N}, Y_{N} \geq Y_{N}^{\mathrm{eq}}$ can be realized at a much lower temperature such as $z>1$ for small values of $h_{N}$. It is found in the right panel of Fig. 2. Thus, the substantial lepton number generation starts at a larger $z$, where $Y_{N}(z)$ is much smaller than $Y_{N}^{\mathrm{eq}}(1)$. This is one of the reasons why the low scale leptogenesis is not so easy in the ordinary seesaw model. The present model could escape this difficulty since the Yukawa coupling $h_{N}$ is irrelevant to both the $N$ production and the neutrino mass generation.

In the above discussion, the washout efficiency $\kappa$ for the generated lepton number asymmetry is not taken into account quantitatively. The lepton number asymmetry could be washed out mainly by the lepton number violating 2-2 scattering such as $\eta \eta \rightarrow \ell_{i} \ell_{j}$ and $\eta \ell_{i} \rightarrow \eta^{\dagger} \bar{\ell}_{j}$, which are mediated by $\Sigma_{\alpha}$, and also the inverse decay of $\Sigma_{\alpha}$ and $N$. Since these processes could be heavily suppressed by the Boltzmann factor at a low temperature region $z \gg 1\left(M_{1} \gg T\right)$, we can take $\kappa \simeq 1$ if the lepton number 

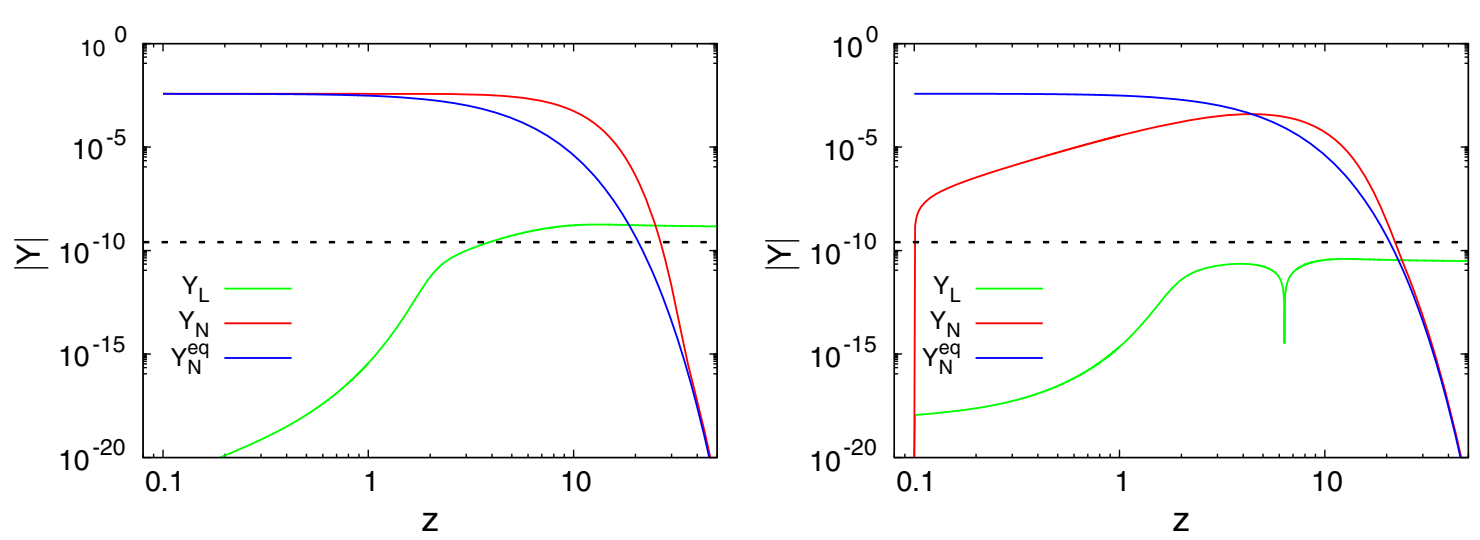

FIG. 4. The evolution of $Y_{L}$ for both initial conditions $Y_{N}\left(z_{i}\right)=Y_{N}^{\mathrm{eq}}\left(z_{i}\right)$ (left panel) and $Y_{N}\left(z_{i}\right)=0$ (right panel). In both panels, the parameters given in (8) are used. Although we use $z_{i}=10^{-1}$ in this analysis, the result is not affected even if $z_{i}$ is taken to be a smaller value, which has been remarked on in Fig. 2. The black dotted lines in each panel represent the required value of $\left|Y_{L}\right|$.

asymmetry is mainly generated in this region. Such a situation is expected to occur in a tiny $h_{N}$ case. On the other hand, if the lepton number asymmetry is generated at a smaller $z$ region such as $z \lesssim 10, \kappa$ could take a smaller value $(\kappa \ll 1)$ there. The above washout processes are proportional to $h_{1,2}^{4}, h_{1,2}^{2}$, and $h_{N}^{2}$, respectively, while the $C P$ asymmetry $\varepsilon_{1,2}$ is proportional to $h_{1,2}^{2}$. Thus, we find that the values of $h_{1,2}$ contained in a restricted region are favored for the generation of the lepton number asymmetry. Such values of $h_{1,2}$ can be realized for a certain range of $\left|\lambda_{5}\right|$ as found from the neutrino mass formula (4) if masses of $\eta$ and $\Sigma_{\alpha}$ are fixed.

To examine a possibility of the low scale leptogenesis suggested above and to estimate the produced baryon number asymmetry quantitatively, we solve the Boltzmann equation for $Y_{L} \equiv Y_{\ell}-Y_{\bar{\ell}}$. In the present model, we can use the equilibrium value $Y_{N}^{\mathrm{eq}}$ as the initial value of $Y_{N}$. It can be realized through the 2-2 scattering of the $\Sigma_{\alpha}$ pair as addressed in the previous part. The Boltzmann equation analyzed here is ${ }^{8}$

$$
\begin{aligned}
\frac{d Y_{L}}{d z}= & \frac{z}{s H\left(M_{N}\right)}\left[\varepsilon\left(\frac{Y_{N}}{Y_{N}^{\mathrm{eq}}}-1\right) \gamma_{N}^{D}\right. \\
& \left.-\frac{2 Y_{L}}{Y_{\ell}^{\mathrm{eq}}}\left\{\sum_{f=N, \Sigma_{\alpha}} \frac{\gamma_{f}^{D}}{4}+\gamma_{\eta \ell}+\gamma_{\eta \eta}\right\}\right],
\end{aligned}
$$

where $Y_{\ell}^{\mathrm{eq}}$ stands for the equilibrium value of leptons which is expressed as $Y_{\ell}^{\mathrm{eq}}=\frac{45}{\pi^{4} g_{*}} ; \gamma_{f}^{D}$ stands for a reaction density for the decay of the fermion $f$; and $\gamma_{\eta \ell}$ and $\gamma_{\eta \eta}$ represent the reaction density for $\eta \ell_{i} \rightarrow \eta^{\dagger} \bar{\ell}_{j}$ and $\eta \eta \rightarrow \ell_{i} \ell_{j}$, respectively.

\footnotetext{
${ }^{8}$ Since the lepton number violation due to the sphaleron is not introduced in this equation, this $Y_{L}$ should be understood as $-Y_{B-L}$.
}

In order to find the behavior of the generated lepton number asymmetry, we use the values listed in Eq. (8) for $h_{N}, M_{N}, M_{1}$, and $M_{2}$. If we fix $M_{\eta}$ and $\lambda_{5}$, the neutrino Yukawa couplings $h_{1,2}$ are determined through Eq. (4) by imposing the neutrino oscillation data. As an example, we fix them at $M_{\eta}=10^{3} \mathrm{GeV}$ and $\left|\lambda_{5}\right|=6 \times 10^{-4}$. ${ }^{9}$ These parameters give the $C P$ asymmetry $|\varepsilon| \simeq 10^{-7}$ for the maximal $C P$ phase. In Fig. 4, the solutions $Y_{N}$ and $\left|Y_{L}\right|$ of Eqs. (9) and (17) are plotted for both initial values $Y_{N}\left(10^{-1}\right)=Y_{N}^{\mathrm{eq}}\left(10^{-1}\right)$ and $Y_{N}\left(10^{-1}\right)=0$. The left panel shows that a sufficient value of $\left|Y_{L}\right|$ for the explanation of the baryon number asymmetry in the Universe can be generated in the former initial value. On the other hand, in the latter case plotted in the right panel, the generated $\left|Y_{L}\right|$ is found not to reach the required value. This result can be easily understood by comparing both panels in Fig. 4, which shows that $Y_{N}$ in the latter case reaches and leaves the equilibrium value at a lower temperature $\left(z_{e} \sim 4\right)$ compared with the former case $\left(z_{e} \sim 1\right)$. It directly results in a smaller value of $\left|Y_{L}\right|$ since it can be approximately estimated from $Y_{L} \simeq \varepsilon \kappa Y_{N}^{\mathrm{eq}}\left(z_{e}\right)$ with the same $\kappa$. This example suggests that the leptogenesis could occur successfully for a rather small mass of the mother fermion in the present model. At a smaller $h_{N}$ region, especially, the sufficient baryon number asymmetry is expected to be obtained, since the sufficiently late decay of $N$ allows almost all the generated lepton number asymmetry to escape the washout $(\kappa \simeq 1)$ and be preserved.

A crucial feature of the leptogenesis is controlled by the coupling constants $h_{N}$ and $\lambda_{5}$ in this model. In order to clarify it, in Fig. 5 we show the dependence of $Y_{B}\left(z_{E W}\right)$ on these parameters by fixing the remaining parameters to some typical values. In the left panel, $Y_{B}\left(z_{E W}\right)$ is plotted for

\footnotetext{
${ }^{9} \mathrm{We}$ consider the $\eta \mathrm{DM}$ here. In that case, we have to note that $\left|\lambda_{5}\right|$ is restricted by the direct DM search experiments as $\left|\lambda_{5}\right|>5 \times 10^{-6}[7,27]$.
} 

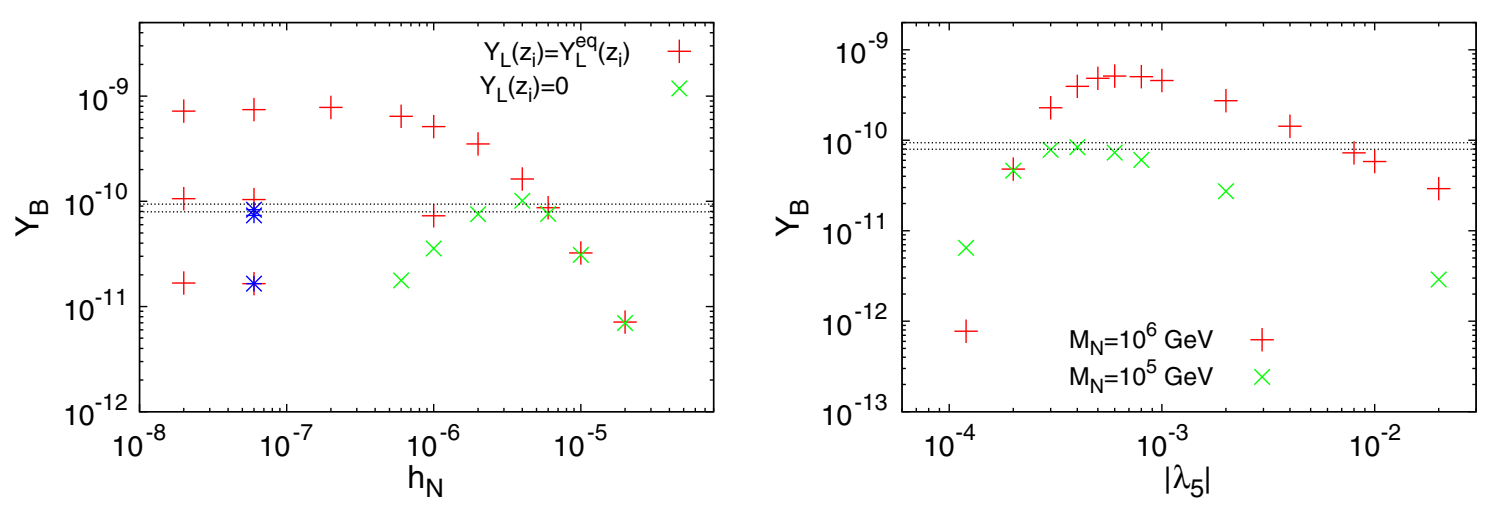

FIG. 5. The dependence of $Y_{B}\left(z_{E W}\right)$ on $h_{N}$ and $\left|\lambda_{5}\right|$. In both panels, $z_{i}=0.1$ is taken and $M_{1,2}$ is assumed to be $M_{\alpha}=10^{\alpha} M_{N}$ except for some cases. The left panel shows the $h_{N}$ dependence of $Y_{B}\left(z_{E W}\right)$ in both initial values of $Y_{N} . M_{N}$ and $\left|\lambda_{5}\right|$ are fixed at $M_{N}=$ $10^{6} \mathrm{GeV}$ and $\left|\lambda_{5}\right|=6 \times 10^{-4}$. At $h_{N}=10^{-6}, 6 \times 10^{-8}$, and $2 \times 10^{-8}$, we plot $Y_{B}\left(z_{E W}\right)$ also for $M_{N}=10^{5}$ and $10^{4} \mathrm{GeV}$ downward. Asterisks at $h_{N}=6 \times 10^{-8}$ display $Y_{B}\left(z_{E W}\right)$ for $M_{N}=10^{4} \mathrm{GeV}, M_{1}=10^{5} \mathrm{GeV}$, and $M_{2}=2 \times 10^{5} \mathrm{GeV}$ by changing $\left|\lambda_{5}\right|$ as $6 \times 10^{-3}, 10^{-4}$, and $6 \times 10^{-4}$ downward. The right panel shows the $\left|\lambda_{5}\right|$ dependence of $Y_{B}\left(z_{E W}\right)$ at $h_{N}=10^{-6}$. The initial condition is fixed at $Y_{N}\left(z_{i}\right)=Y_{N}^{\mathrm{eq}}\left(z_{i}\right)$.

various values of $h_{N}$ by using both initial values $Y_{N}\left(10^{-1}\right)=Y_{N}^{\mathrm{eq}}\left(10^{-1}\right)$ and $Y_{N}\left(10^{-1}\right)=0$. As discussed in Eq. (16), the coupling $h_{N}$ determines a period where $N$ is in the out-of-equilibrium state. Taking this into account, the $Y_{B}\left(z_{E W}\right)$ behavior shown in this panel can be explained as follows. In the case $Y_{N}\left(10^{-1}\right)=Y_{N}^{\mathrm{eq}}\left(10^{-1}\right)$, the smaller $h_{N}$ makes the substantial $N$ decay be delayed until a low temperature where the washout processes are frozen out. As a result, almost all the lepton number asymmetry generated through the $N$ decay is transformed to $Y_{B}$ independently of the $h_{N}$ value. It explains the almost constant behavior of $Y_{B}\left(z_{E W}\right)$ at a small $h_{N}$ region such as $h_{N} \lesssim 2 \times 10^{-7}$. In the region $h_{N} \lesssim 4 \times 10^{-6}$, the sufficient $Y_{B}$ can be obtained for the $Y_{N}\left(10^{-1}\right)=Y_{N}^{\mathrm{eq}}\left(10^{-1}\right)$ case, since the washout due to the inverse decay of $N$ is suppressed. On the other hand, in the $Y_{N}\left(10^{-1}\right)=0$ case, $Y_{N}>Y_{N}^{\mathrm{eq}}$ tends to be realized at a later period such as $z \gg 1$. For a such region of $z, Y_{N}^{\mathrm{eq}}$ is too small to generate the sufficient $Y_{B}\left(z_{E W}\right)$. In the region $h_{N} \gtrsim 5 \times 10^{-6}, Y_{N}>Y_{N}^{\mathrm{eq}}$ starts at $z \sim 1$ commonly for both initial values, and then the same $Y_{B}\left(z_{E W}\right)$ value is obtained for them. Although $Y_{N}$ could be sufficiently large in this case, the washout due to the inverse decay of $N$ is effective for this range of $h_{N}$ and then it is difficult for $Y_{B}\left(z_{E W}\right)$ to reach a required value. Here, it may be useful to note that the required $Y_{B}\left(z_{E W}\right)$ could be obtained for a suitable value of $h_{N}$ even in a situation $Y_{N}\left(10^{-1}\right)=0$ and $M_{N}<10^{8} \mathrm{GeV}$, as found in the left panel. It is considered to be caused by the hybrid nature of the model which makes the $C P$ asymmetry $\varepsilon$ larger compared with the pure scotogenic type I seesaw model [7].

In the right panel of Fig. 5, $Y_{B}\left(z_{E W}\right)$ is plotted for various values of $\left|\lambda_{5}\right|$ for two values of $M_{N}$. In this calculation, we choose $h_{N}=10^{-6}$ and then the washout is considered to be mainly caused by $\Sigma_{\alpha}$. The figure shows that the $\left|\lambda_{5}\right|$ values included in a restricted region can generate a sufficient amount of $Y_{B}\left(z_{E W}\right)$. The coupling $\lambda_{5}$ determines both magnitudes of the $C P$ asymmetry $\varepsilon$ and the washout efficiency $\kappa$ through the neutrino Yukawa couplings $h_{1,2}$. A larger $\left|\lambda_{5}\right|$ gives the smaller $h_{1,2}$ under the constraint of the neutrino oscillation data. It explains the $Y_{B}\left(z_{E W}\right)$ behavior presented in this figure.

Another interesting issue of the model is what is a lower bound of $M_{N}$ for which the required value of $Y_{B}\left(z_{E W}\right)$ can be obtained. At $h_{N}=6 \times 10^{-8}$ in the left panel of Fig. 5, $Y_{B}\left(z_{E W}\right)$ is plotted by asterisks for $M_{N}=10^{4} \mathrm{GeV}$, $M_{1}=10^{5} \mathrm{GeV}$, and $M_{2}=2 \times 10^{5} \mathrm{GeV}$ changing the value of $\left|\lambda_{5}\right|$ downward as $6 \times 10^{-5}, 10^{-4}, 6 \times 10^{-4}$. In order to show what causes the difference among the cases with $M_{N}=10^{4} \mathrm{GeV}$ and $h_{N}=6 \times 10^{-8}$, we list parameters relevant to the leptogenesis in Table I. This suggests that the lower bound of $M_{N}$ could be $10^{4} \mathrm{GeV}$ at least in the present model. ${ }^{10}$ If the relevant parameters in the model are fixed at appropriate values which can realize $|\varepsilon| \gtrsim 10^{-7}$ and suppress the washout due to $\Sigma_{\alpha}$ simultaneously at least for a sufficiently small $h_{N}$, the low scale leptogenesis could be allowed in this model in a consistent way with the neutrino mass generation, the DM abundance, and also the inflation. We need no serious tuning for them even in that case.

Finally, we remark on the signatures in the collider experiment caused by the present low scale leptogenesis. Collider phenomenology expected for the triplet fermions has been discussed extensively in [28]. Following it, any promising signature of the triplet fermions cannot be expected in the collider physics at least in the near future,

\footnotetext{
${ }^{10}$ The possibility of low scale leptogenesis in the scotogenic type I seesaw has been intensively studied in [24]. They concluded $M_{N} \gtrsim 10^{4} \mathrm{GeV}$ for the successful leptogenesis, just assuming $N$ is in thermal equilibrium initially. Although we do not exhaust the parameter space, a similar bound of $M_{N}$ is obtained in the present model.
} 
TABLE I. The $C P$ asymmetry $\varepsilon$ and the baryon number asymmetry $Y_{B}\left(z_{E W}\right)$ for several $M_{1,2}$ and $\left|\lambda_{5}\right| . M_{N}$ and $h_{N}$ are fixed at $10^{4} \mathrm{GeV}$ and $6 \times 10^{-8}$, respectively. The Yukawa coupling constants $h_{1,2}$ for $\Sigma_{1,2}$ are determined by the neutrino oscillation data.

\begin{tabular}{lccccc}
\hline \hline$M_{1}(\mathrm{GeV})$ & $M_{2}(\mathrm{GeV})$ & $\left|\lambda_{5}\right|$ & $h_{1}$ & $h_{2}$ & $|\varepsilon|$ \\
\hline $2 \times 10^{4}$ & $4 \times 10^{4}$ & $6 \times 10^{-5}$ & $4.2 \times 10^{-3}$ & $1.8 \times 10^{-3}$ & $2.4 \times 10^{-7}$ \\
$2 \times 10^{4}$ & $4 \times 10^{4}$ & $10^{-4}$ & $3.2 \times 10^{-3}$ & $1.4 \times 10^{-3}$ & $1.4 \times 10^{-7}$ \\
$10^{5}$ & $2 \times 10^{5}$ & $6 \times 10^{-5}$ & $7.3 \times 10^{-3}$ & $3.2 \times 10^{-3}$ & $1.6 \times 10^{-7}$ \\
$10^{5}$ & $2 \times 10^{5}$ & $10^{-4}$ & $5.6 \times 10^{-3}$ & $2.5 \times 10^{-3}$ & $9.5 \times 10^{-8}$ \\
$10^{5}$ & $10^{6}$ & $6 \times 10^{-5}$ & $7.3 \times 10^{-3}$ & $6.3 \times 10^{-3}$ & $1.6 \times 10^{-7}$ \\
$10^{5}$ & $10^{6}$ & $10^{-4}$ & $5.6 \times 10^{-3}$ & $4.8 \times 10^{-3}$ & $8.4 \times 10^{-11}$ \\
\hline \hline
\end{tabular}

since their masses should be larger than $O\left(10^{4}\right) \mathrm{GeV}$ for successful leptogenesis. On the other hand, even if the signatures of inert doublet scalars $\eta$ are discovered, it seems to be difficult to distinguish the scotogenic type III model from the scotogenic type I model.

\section{SUMMARY}

The scotogenic type III seesaw model is an interesting model which can link the neutrino mass generation and the existence of DM. Unfortunately, it cannot explain the baryon number asymmetry in the Universe through leptogenesis. Since heavy fermions in the model are triplets of $S U(2)$ and then have the gauge interaction, they are kept in thermal equilibrium until the electroweak scale. As a consequence, they cannot generate the lepton number asymmetry through the out-of-equilibrium decay.

We proposed a simple extension of the model by introducing a singlet fermion so as to incorporate successful leptogenesis. Since this singlet fermion could be irrelevant to the neutrino mass generation by assuming its Yukawa coupling constants are very small, its out-ofequilibrium decay could be possible even if it is not so heavy. If its thermal equilibrium could be prepared not through its Yukawa couplings but through other interactions, the leptogenesis caused by its decay at a low temperature region could explain the required baryon number asymmetry. As such a process, we supposed the singlet fermion pair production caused by the pair annihilation of the triplet fermions which are produced in the inflaton decay. Since the triplet fermions are in thermal equilibrium at an early stage, the singlet fermions could reach thermal equilibrium at a high temperature where its equilibrium number density takes a large value. Several parameter dependences of this leptogenesis were clarified in detail. We also showed that the required baryon number asymmetry could be generated even for the small mass of the singlet fermion like $O\left(10^{4}\right) \mathrm{GeV}$ as long as the relevant parameters have suitable values. The scenario might be applicable for the low scale leptogenesis in other models for the neutrino mass, the DM, and the inflation.

\section{ACKNOWLEDGMENTS}

This work is partially supported by a Ministry of Education, Culture. Sports, Science and Technology Grant-in-Aid for Scientific Research on Innovative Areas (Grant No. 26104009) and Japan Society for Promotion of Science, a Grant-in Aid for Scientific Research (C) (Grant No. 18K03644).
[1] M. Fukugita and T. Yanagida, Phys. Lett. B 174, 45 (1986).

[2] M. Plümacher, Nucl. Phys. B530, 207 (1998); W. Buchmüller and M. Plümacher, Int. J. Mod. Phys. A 15, 5047 (2000); W. Buchmüller, P. Di Bari, and M. Plümacher, Phys. Lett. B 547, 128 (2002); Nucl. Phys. B643, 367 (2002); B665, 445 (2003); G. F. Giudice, A. Notari, M. Raidal, A. Riotto, and A. Struma, Nucl. Phys. B685, 89 (2004); W. Buchmüller, R. D. Peccei, and T. Yanagida, Annu. Rev. Nucl. Part. Sci. 55, 311 (2005).

[3] V. A. Kuzmin, V. A. Rubakov, and M. E. Shapshnikov, Phys. Lett. 155B, 36 (1985).

[4] P. Minkowski, Phys. Lett. 67B, 421 (1977); M. GellMann, P. Ramond, and R. Slansly, in Supergravity, edited by D. Freedman and P. Van Nieuwenhuizen (North Holland, Amsterdam, 1979), p. 315; T. Yanagida, Prog. Theor. Phys. 64, 1103 (1980); R. N. Mohapatra and G. Senjanović, Phys. Rev. Lett. 44, 912 (1980).

[5] S. Davidson and A. Ibarra, Phys. Lett. B 535, 25 (2002).

[6] M. Flanz, E. A. Pascos, and U. Sarkar, Phys. Lett. B 345, 248 (1995); L. Covi, E. Roulet, and F. Vissani, Phys. Lett. B 384, 169 (1996); E. Akhmedov, M. Frigerio, and A. Yu Smirnov, J. High Energy Phys. 09 (2003) 021; C. H. Albright and S. M. Barr, Phys. Rev. D 69, 073010 (2004); T. Hambye, J. March-Russell, and S. W. West, J. High Energy Phys. 07 (2004) 070; A. Pilaftsis and E. J. Underwood, Nucl. Phys. B692, 303 (2004); Phys. Rev. D 72, 113001 (2005). 
[7] S. Kashiwase and D. Suematsu, Phys. Rev. D 86, 053001 (2012); Eur. Phys. J. C 73, 2484 (2013).

[8] E. Ma, Phys. Rev. D 73, 077301 (2006); Mod. Phys. Lett. A 21, 1777 (2006).

[9] J. Kubo, E. Ma, and D. Suematsu, Phys. Lett. B 642, 18 (2006); D. Suematsu, Eur. Phys. J. C 56, 379 (2008); D. Aristizabal Sierra, J. Kubo, D. Restrepo, D. Suematsu, and O. Zapata, Phys. Rev. D 79, 013011 (2009); D. Suematsu, T. Toma, and T. Yoshida, Phys. Rev. D 79, 093004 (2009); S. Kashiwase and D. Suematsu, Eur. Phys. J. C 76, 117 (2016); M. Lindner, M. Platscher, and F. S. Queiroz, Phys. Rep. 731, 1 (2018).

[10] D. Suematsu, Eur. Phys. J. C 72, 1951 (2012); S. Baumholzer, V. Brdar, and P. Schwaller, J. High Energy Phys. 08 (2018) 067.

[11] E. Ma and D. Suematsu, Mod. Phys. Lett. A 24, 583 (2009).

[12] D. Suematsu, arXiv:1906.10849.

[13] T. Hambye, Y. Lin, A. Notari, M. Papucci, and A. Strumia, Nucl. Phys. B695, 169 (2004); D. A. Sierra, J. F. Kamenik, and M. Nemevsek, J. High Energy Phys. 10 (2010) 036.

[14] E. Ma, Phys. Lett. B 625, 76 (2005); W. Fischler and R. Flauger, J. High Energy Phys. 09 (2008) 020.

[15] R. Barbieri, L. J. Hall, and V. S. Rychkov, Phys. Rev. D 74, 015007 (2006); M. Cirelli, N. Fornengo, and A. Strumia, Nucl. Phys. B753, 178 (2006); L. L. Honorez, E. Nezri, J. F. Oliver, and M. H. G. Tytgat, J. Cosmol. Astropart. Phys. 02 (2007) 028; Q.-H. Cao and E. Ma, Phys. Rev. D 76, 095011 (2007); T. Hambye, F.-S. Ling, L. L. Honorez, and J. Roche, J. High Energy Phys. 07 (2009) 090; S. Andreas, M. H. G. Tytgat, and Q. Swillens, J. Cosmol. Astropart. Phys. 04 (2009) 004; E. Nezri, M. H. G. Tytday, and G. Vertongen, J. Cosmol. Astropart. Phys. 04 (2009) 014; L. L. Honorez, J. Cosmol. Astropart. Phys. 01 (2011) 002.

[16] M. Hirsch, R. A. Lineros, S. Morosi, J. Palacio, N. Rojas, and J. W. E. Valle, J. High Energy Phys. 10 (2013) 149; P. Rocha-Moran and A. Vicente, J. High Energy Phys. 07 (2016) 078.
[17] F. Bezrukov and M. E. Shapshnikov, Phys. Lett. B 659, 703 (2008); F. L. Bezrukov, A. Magnin, and M. Shaposhnikov, Phys. Lett. B 675, 88 (2009).

[18] A. De Simone, M. P. Hertzberg, and F. Wilczek, Phys. Lett. B 678, 1 (2009); G. F. Guidice and H. M. Lee, Phys. Lett. B 694, 294 (2011); F. Bezrukov, A. Magnin, M. Shaposhnikov, and S. Sibiryakov, J. High Energy Phys. 01 (2011) 016; R. N. Lerner and J. McDonald, Phys. Rev. D 83, 123522 (2011); J. Elias-Miró, J. R. Espinosa, G. F. Giudice, G. Isidori, A. Riotto, and A. Strumia, Phys. Lett. B 709, 222 (2012); X. Calmet and R. Casadio, Phys. Lett. B 734, 17 (2014).

[19] D. Suematsu, Phys. Rev. D 85, 073008 (2012); R. H. S. Budhi, S. Kashiwase, and D. Suematsu, Phys. Rev. D 90, 113013 (2014); 93, 013022 (2016); Phys. Lett. B 749, 603 (2015); D. Suematsu, Phys. Lett. B 760, 538 (2016).

[20] E. W. Kolb and S. Wolfram, Nucl. Phys. B172, 224 (1980); E. W. Kolb and M. S. Turner, The Early Universe (AddisonWesley, Redwood City, CA, 1990).

[21] M. Luty, Phys. Rev. D 45, 455 (1992); M. Plümacher, Nucl. Phys. B530, 207 (1998).

[22] S. Yu Khlebnikov and M.E. Shapshnikov, Nucl. Phys. B308, 885 (1988); J. A. Harvey and M. S. Turner, Phys. Rev. D 42, 3344 (1990).

[23] A. Pilaftsis, Phys. Rev. D 56, 5431 (1997).

[24] T. Hugle, M. Platscher, and K. Schmitz, Phys. Rev. D 98, 023020 (2018); D. Borah, P. S. B. Dev, and A. Kumar, Phys. Rev. D 99, 055012 (2019).

[25] J. Kubo and D. Suematsu, Phys. Lett. B 643, 336 (2006); D. Suematsu, T. Toma, and T. Yoshida, Phys. Rev. D 79, 093004 (2009).

[26] M. Tanabashi et al. (Particle Data Group), Phys. Rev. D 98, 030001 (2018).

[27] Y. Cui, D. E. Marrissey, D. Poland, and L. Randall, J. High Energy Phys. 05 (2009) 076; C. Arina, F.-S. Ling, and M. H. G. Tytgat, J. Cosmol. Astropart. Phys. 10 (2009) 018 .

[28] R. Francesschini, T. Hambye, and A. Strumia, Phys. Rev. D 78, 033002 (2008). 